\title{
Invasive-Invaded System of Non-Lipschitz Porous Medium Equations with Advection
}

\author{
October 7, 2021
}

José Luis Díaz Palencia

joseluis.diaz@ufv.es

Universidad Francisco de Vitoria

Escuela Politécnica Superior, Universidad Francisco de Vitoria, Ctra. Pozuelo-Majadahonda Km 1,800, 28223, Pozuelo de Alarcón, Madrid

\begin{abstract}
This work provides analytical results towards applications in the field of invasive-invaded systems modelled with non-linear diffusion and with advection. The results focus on showing regularity, existence and uniqueness of weak solutions using the condition of a non-linear slightly positive parabolic operator and the reaction-absorption monotone properties. The coupling in the reaction-absorption terms, that characterizes the species interaction, impedes the formulation of a global comparison principle that is shown to exist locally. Additionally, the present work provides analytical solutions obtained as selfsimilar minimal and maximal profiles. A propagating diffusive front is shown to exist until the invaded specie notes the existence of the invasive. When the desertion of the invaded starts, the diffusive front vanishes globally and the non-linear diffusion concentrates only on the propagating tail which exhibits finite speed. Finally, the invaded specie is shown to exhibit an exponential decay along a characteristic curve. Such exponential decay is not trivial in the non-linear diffusion case and confirms that the invasive continues to feed on the invaded during the desertion.
\end{abstract}

Keywords: non-linearity, reaction, absorption, coupled system, diffusion, porous media.

AMS Subject Classification: 35K55, 35K57, 35K59, 35K65

\section{Introduction}

According to the Convention of Biological Diversity (p. 1, Ch. 1 [23]), the biological invasion is defined as "...those alien species which threaten ecosystems, habitats or species". The problem analyzed can be understood within the perspective of an invasive specie (predator) that invades a region or space previously inhabited by the invaded specie (prey).

Predator-prey models are an active source of research. In [5], different forms of functional responses are provided for modelling the predator-prey dynamic. The cited work considers the harvesting in the predator and the density-dependent mortality in the prey to assess the Hopf bifurcation in the proximity of the equilibrium point. In addition, the Hopf bifurcation method has been followed in [19] to study a delayed density-dependent predator-prey system with BeddingtonDeAngelis functional response. The periodic dynamic in the solutions has been shown to exist as a consequences of stochastic disturbance for a Holling II functional response [34]. Similar stability and bifurcation methods have been used in [24] for a predator-prey model subject to the Allee effect with a discrete-time Holling type-IV functional response. The mentioned methods have been used as well in [20] to model an infected predator that consumes the prey according to Holling type-II response. 
Alternatively, the solving methods exposed along this work are based on explicit analytical solutions that consider the spatial propagation features in the form of a non-linear diffusion with advection. The spatial operator is considered together with non-Lipschitz reaction-absorption terms. Such terms are introduced based on the following proposed dynamic:

Consider that the invasive specie acts qualitatively fast so that the invaded specie reproduction rate is negligible. As the invasive specie proliferates over time, the temporal ratio (considered positive) decreases due to the vanishing of invaded specie on which the invasive specie feeds on. This behavior is intended to be modelled as a reaction term of the form:

$$
u_{t}=v^{n}, \quad n \in(0,1),
$$

to account for the saturation in the medium by the invasive specie when the invaded one tends to zero. Note that $v^{n}$ is a non-Lipschitz function.

The invaded specie time vanishing rate reads:

$$
v_{t}=-u^{p} \quad p \in(0,1) .
$$

The introduction of the invasive agent initially $\left(u_{0}>0\right)$ makes $v$ to decrease relatively fast and decelerates as long as $u$ increases in accordance with the convex behaviour in $-u^{p}$.

An advection term is proposed to account for possible forced movement in the media. The interaction of the advection through the vector $c$ has the intention of modelling the desertion behaviour in the invaded specie, so that the runaway is oriented in the direction of $c$ and toward spatial areas not previously populated (represented by $c \cdot \nabla v$ ). In response to the invaded specie desertion, the invasive specie will follow an advection process given by the orientation $c$ towards the same non-populated areas (represented by $c \cdot \nabla u$ ).

In addition, a non-linear diffusion is considered to model the random movement of both species. The invasive-invaded system with classical order two diffusion and with Lipsthitz reaction and absorption is a well known problem (Ch.4 in [4]). Recently, Díaz and Fernández [15] have proposed a set of equations to study a predator-prey parabolic model with convection and a non-Lipschitz reaction-absorption. As an alternative to the classical linear order two diffusion, the Porous Medium Equation introduces concepts such as the finite speed of propagation in the invaded specie which permits to account for a further accurate modelization. A non-linear diffusion model was proposed initially by Keller and Segel [22] to study the cells movement by chemotaxis:

$$
\begin{array}{cc}
u_{t}=\nabla \cdot(d(u) u-\chi(v) u \nabla u) & x \in \Omega, t>0 \\
v_{t}=d_{v} \Delta v-u v & x \in \Omega, t>0
\end{array}
$$

where $u$ represents the cell density and $v$ the chemical concentration. Note that $d(u)$ is the media diffusivity and $\chi(v)$ the distribution of chemical agent to which the cells are sensitive. The Keller and Segel model was progressively extended to incorporate particular kinetic dynamics in the form of reaction and absorption terms [3], [11], [32], [29] with different structures complying with a regularity criteria including the Lipschtiz condition. The problem discussed in [28] was proposed as an integro-differential system of equations with pressure effects to predict the behaviour of cancerous cells, as an invasive specie, spreading with a non-linear diffusion over healthy cells in a closed organ.

The use of non-linear diffusion is an oblique topic and has been used in other interesting applications where numerical and purely analytical approaches have been followed. Such nonlinear diffusion allows to model accurately physical phenomena in which porosity is a governing parameter. The Darcy law involving nanofluids has been considered to derive numerical solutions by the Successive Local Linearization Method [26]. The authors show the different solution profiles obtained after the numerical exercise where the exponential decay can be perceived. Such an exponential decay is immediate in the linear diffusion case, nonetheless for the non-linear diffusion further analytical assessments shall be done. The effect of porosity in a partial slip for a peristaltic transport in a Jeffrey fluid has been investigated in [19]. In addition [10] employs a non-linear diffusion to improve the accuracy in simulating potential coagulation in an electromagnetic blood flow in annular vessel geometries. In all the cited cases, the finite speed of propagation defines a diffusive front. This is a common behaviour to all porous medium equations and shall be characterized for the problem discussed along the present work. 
The proposed problem (named as $P$ ) reads:

$$
\begin{gathered}
u_{t}=\Delta u^{m}+c \cdot \nabla u+v^{n}, \\
v_{t}=\Delta v^{m}+c \cdot \nabla v-u^{p}, \\
n, p \in(0,1), \\
u_{0}(x), v_{0}(x)>0 \in L^{1}\left(\mathbb{R}^{d}\right) \cap L^{\infty}\left(\mathbb{R}^{d}\right) .
\end{gathered}
$$

The study of solutions for a Porous Medium Equation with advection and no reaction has been widely analyzed in [33]. Nonetheless, the present paper considers the effect of the non-Lipschitz reaction and absorption for which the coupling monotone behaviour induces a different analytical treatment compared to that in [33].

This work begins by showing some regularity results together with uniqueness of solutions. The degeneracy of the diffusivity leads to consider weak solutions defined upon a test function $\phi \in C^{\infty}\left(\mathbb{R}^{d}\right)$. For this purpose, the monotony properties of the quasi positive operator and of the reaction-absorption are employed with a proper definition of regular sequences of solutions. A comparison principle between solutions does not hold in general due to the coupling in the reaction and absorption terms, nonetheless, the most general conditions for existence of such comparison principle are provided. Afterwards, profiles of maximal and minimal solutions are shown to exist and analytically obtained. Finally, the finite propagation and the exponential decay for $v$ are shown in the proximity of the null solution, i. e. when $v \rightarrow \epsilon \rightarrow 0^{+}$. The finite propagation is proved with a maximal solution so that any other lower solution exhibits finite propagation as well. The existence of an exponential decay is shown based on an asymptotic separation of variables leading to a transport equation with solutions along a characteristic curve precisely determined. The transport equation elucidates the existence of a trajectory (along characteristics) run by the invaded specie during its desertion. This means that the homogenizing effect of the diffusive front is lost. In addition, the invasive is capable of concentrating efforts in the runaway direction $c$ so that the invaded specie decreases exponentially.

\section{Regularity, existence, uniqueness and comparison of solu- tions}

Consider the test function $\phi \in C^{\infty}\left(\mathbb{R}^{d}\right)$ such tat for $0<\tau<t<T$ :

$$
\begin{aligned}
\int_{\mathbb{R}^{d}} u(t) \phi(t) & =\int_{\mathbb{R}^{d}} u(\tau) \phi(\tau)+\int_{\tau}^{t} \int_{\mathbb{R}^{d}}\left[u \phi_{t}+u^{m} \Delta \phi+c \cdot \nabla \phi u+v^{n} \phi\right] d s, \\
\int_{\mathbb{R}^{d}} v(t) \phi(t) & =\int_{\mathbb{R}^{d}} v(\tau) \phi(\tau)+\int_{\tau}^{t} \int_{\mathbb{R}^{d}}\left[v \phi_{t}+v^{m} \Delta \phi+c \cdot \nabla \phi v-u^{p} \phi\right] d s .
\end{aligned}
$$

Consider $r>r_{0}>0$, the following uniformly parabolic quasilinear set of equations is defined (named $P^{\phi}$ ):

$$
\begin{aligned}
& u \phi_{t}+u^{m} \Delta \phi+c \cdot \nabla \phi u+v^{n} \phi=0 \\
& v \phi_{t}+v^{m} \Delta \phi+c \cdot \nabla \phi v-u^{p} \phi=0
\end{aligned}
$$

in $B_{r} \times[0, T]$, with the following set of boundary and initial conditions:

$$
\begin{aligned}
& (\nabla \phi+u c) \cdot \nu=0 \\
& (\nabla \phi+v c) \cdot \nu=0 .
\end{aligned}
$$

over $\partial B_{r} \times[0, T]$, where $\nu$ is the outer unitary normal vector in $\partial B_{r}$ and

$$
\begin{aligned}
& u(x, 0)=u_{0}(x), \\
& v(x, 0)=v_{0}(x) .
\end{aligned}
$$

initially on $B_{r}$.

Let consider the following truncation:

$$
u_{\epsilon}^{m}=\left[\begin{array}{ccc}
u^{m} & \text { when } & |u|<\epsilon \\
\epsilon^{m} & \text { when } & |u| \geq \epsilon
\end{array}\right],
$$




$$
u_{\epsilon}=\left[\begin{array}{l}
u \text { when }|u|<\epsilon \\
\epsilon \text { when }|u| \geq \epsilon
\end{array}\right] .
$$

The same truncation is defined analogously for $v$. The problem $P^{\phi}$ can be reformulated to provide the problem $P_{\epsilon}^{\phi}$ as:

$$
\begin{aligned}
& \mathrm{u}_{\epsilon} \phi_{t}+u_{\epsilon}^{m} \Delta \phi+c \cdot \nabla \phi u_{\epsilon}+v_{\epsilon}^{n} \phi=0, \\
& \mathrm{v}_{\epsilon} \phi_{t}+v_{\epsilon}^{m} \Delta \phi+c \cdot \nabla \phi v_{\epsilon}-u_{\epsilon}^{p} \phi=0,
\end{aligned}
$$

with the associated set of boundary and initial conditions (2.4) and (2.5).

The problem $P_{\epsilon}^{\phi}$ has existence and uniqueness of solutions for any value of the parameter $\epsilon$ based on the monotone properties of the forcing terms [25]. For this purpose, note that the forcing part $\phi$ is a monotone increasing (with $\phi$ ) function and $-\phi$ is a monotone decreasing function.

Theorem 1. Consider that $u=u_{\epsilon}$ and $v=v_{\epsilon}$, with initial data $u_{0}(x), v_{0}(x) \in L^{1}\left(\mathbb{R}^{d}\right) \cap L^{\infty}\left(\mathbb{R}^{d}\right)$, solve the problem $P$ when $\epsilon>>1$ and $r \rightarrow \infty$. Then, the set of solutions $u(x, t), v(x, t)$ are bounded for all $(x, t) \in \mathbb{R}^{d} \times[0, \infty)$.

Proof. Consider $\eta>0, \eta \in \mathbb{R}$ and sufficiently small so that the following cut off function is defined [13]:

$$
\begin{array}{cc}
\psi_{\eta} \in C_{0}^{\infty}\left(\mathbb{R}^{d}\right), & 0 \leq \psi_{\eta} \leq 1, \\
\psi_{\eta}=1 \text { in } B_{r-\eta}, & \psi_{\eta}=0 \text { in } \mathbb{R}^{d}-B_{r-\eta},
\end{array}
$$

so that

$$
\begin{aligned}
& \left|\nabla \psi_{\eta}\right| \leq \frac{c_{a}}{\eta}, \\
& \left|\Delta \psi_{\eta}\right| \leq \frac{c_{a}}{\eta^{2}} .
\end{aligned}
$$

After multiplication of (2.3) by $\psi_{\eta}$ and integrating in $B_{r} \times[\tau, T]$ :

$$
\begin{aligned}
& \int_{\tau}^{t} \int_{B_{r}} u \phi_{t} \psi_{\eta}+\int_{\tau}^{t} \int_{B_{r}} u^{m} \Delta \phi \psi_{\eta}+\int_{\tau}^{t} \int_{B_{r}} c \cdot \nabla \phi u \psi_{\eta}+\int_{\tau}^{t} \int_{B_{r}} v^{n} \phi \psi_{\eta}=0, \\
& \int_{\tau}^{t} \int_{B_{r}} v \phi_{t} \psi_{\eta}+\int_{\tau}^{t} \int_{B_{r}} v^{m} \Delta \phi \psi_{\eta}+\int_{\tau}^{t} \int_{B_{r}} c \cdot \nabla \phi v \psi_{\eta}-\int_{\tau}^{t} \int_{B_{r}} u^{p} \psi_{\eta} \phi=0 .
\end{aligned}
$$

The integrals for the diffusion and advection terms read:

$$
\begin{gathered}
\int_{\tau}^{t} \int_{B_{r}} c \cdot \nabla \phi u \psi_{\eta}=-\int_{\tau}^{t} \int_{B_{r}} u \phi c \cdot \nabla \psi_{\eta}, \\
\int_{\tau}^{t} \int_{B_{r}} u^{m} \Delta \phi \psi_{\eta}=-\int_{\tau}^{t} \int_{B_{r}} u^{m} \nabla \phi \cdot \nabla \psi_{\eta} .
\end{gathered}
$$

Then:

$$
\begin{aligned}
& \int_{\tau}^{t} \int_{B_{r}} u \phi_{t} \psi_{\eta}+\int_{\tau}^{t} \int_{B_{r}} v^{n} \phi \psi_{\eta}=\int_{\tau}^{t} \int_{B_{r}} u \phi c \cdot \nabla \psi_{\eta}+\int_{\tau}^{t} \int_{B_{r}} u^{m} \nabla \phi \cdot \nabla \psi_{\eta}, \\
& \int_{\tau}^{t} \int_{B_{r}} v \phi_{t} \psi_{\eta}-\int_{\tau}^{t} \int_{B_{r}} u^{p} \psi_{\eta} \phi=\int_{\tau}^{t} \int_{B_{r}} v \phi c \cdot \nabla \psi_{\eta}+\int_{\tau}^{t} \int_{B_{r}} v^{m} \nabla \phi \cdot \nabla \psi_{\eta} .
\end{aligned}
$$

The intention is to show that the left hand integrals in (2.14) are finite which is equivalent to search for the $L^{\infty}$ norm on the right hand side terms.

For some large $r>>r_{0}>1[13]$ :

$$
\int_{\tau}^{t} u^{m} \leq c_{1}(\tau) r^{\frac{2 m}{m-1}}
$$

and

$$
\int_{\tau}^{t} u \leq c_{2}(\tau) r^{\frac{2}{m-1}}
$$

Then:

$$
\int_{\tau}^{t} \int_{B_{r}} u^{m} \nabla \phi \cdot \nabla \psi_{\eta} \leq \int_{B_{r}} c_{1}(\tau) r^{\frac{2 m}{m-1}}|\nabla \phi| \frac{c_{a}}{\eta} \leq c_{1}(\tau) c_{a} \int_{B_{r}} r^{\frac{2 m}{m-1}-1}|\nabla \phi| .
$$




$$
\int_{\tau}^{t} \int_{B_{r}} u \phi c \cdot \nabla \psi_{\eta} \leq c c_{2}(\tau) c_{a} \int_{B_{r}} r^{\frac{2}{m-1}-1} \phi .
$$

Next, consider a test function $\phi$ of the form:

$$
\phi(x, s)=e^{g(s)}\left(1+r^{2}\right)^{-\beta},
$$

where $g(s)>0$ for $0<s<t, g(t)=0$ and $\beta$ shall be chosen to ensure the converge of $(2.17)$ and $(2.18)$ as $r \rightarrow \infty$. Then $(2.17)$ is:

$$
c_{1}(\tau) c_{a} \int_{B_{r \rightarrow \infty}} r^{\frac{2 m}{m-1}-1} 2 \beta e^{g(s)} r^{-2 \beta-1},
$$

and (2.18):

$$
c c_{2}(\tau) c_{a} \int_{B_{r}} r^{\frac{2}{m-1}-1} e^{g(s)} r^{-2 \beta} .
$$

The convergence is ensured for

$$
\beta=\frac{1}{m-1}
$$

Coming to (2.14):

$$
\begin{gathered}
\int_{\tau}^{t} \int_{B_{r \rightarrow \infty}} u \phi_{t} \psi_{\eta}+\int_{\tau}^{t} \int_{B_{r} r \rightarrow \infty} v^{n} \phi \psi_{\eta} \leq c c_{2}(\tau) c_{a} e^{g(s)}, \\
\int_{\tau}^{t} \int_{B_{r \rightarrow \infty}} v \phi_{t} \psi_{\eta}-\int_{\tau}^{t} \int_{B_{r \rightarrow \infty}} u^{p} \psi_{\eta} \phi \leq c c_{2}(\tau) c_{a} e^{g(s)} .
\end{gathered}
$$

As both integrals are finite in $\tau<s<t<T$, it is possible to conclude on the theorem postulation about the boundness of solutions in $\mathbb{R}^{d} \times[0, \infty]$.

Before entering into the existence discussion, the following definitions are provided:

Definition 1. Given a function $f \in C^{2+\gamma, 1+\gamma / 2}\left(\mathbb{R}^{d} \times(0, T)\right), f^{m} \in C^{2+\gamma}\left(\mathbb{R}^{d} \times(0, T)\right)$ for $\gamma>0$, the following linear operator is defined:

$$
L f=f_{t}-\Delta f^{m}-c \cdot \nabla f .
$$

Note that the diffusivity terms in $P$ for each equation are:

$$
D(u)=u^{m-1}, \quad D(v)=v^{m-1} .
$$

which are positive in a local ball $B_{R} \in \mathbb{R}^{d}$ and arbitrary small $T$ provided $u_{0}>0$ and $v_{0}>0$. The potential application of Problem $P$ to biological problems suggests:

$$
u>0 ; v=\max \{v, \epsilon\}
$$

in $\mathbb{R}^{d} \times[0, \infty]$ and where $\epsilon \rightarrow 0^{+}$. Such positivity conditions prevent the degeneracy due to the non-linear diffusion in $u$ while $v$ may exhibit finite propagation. The previous works in [7], [8], [9] and [33] showed the existence of solutions for an aggregation equation with no reaction-absorption term as it is the case in $u$ when $v$ approaches $\epsilon$. Nonetheless, the lack of a Lipschitz condition makes the equation in $u$ to lose the regularity introduced by the positive diffusivity. Consequently, the existence of solutions is shown based on the use of the cited references and considering the specific existence questions introduced by the non-Lipschitz reaction and absorption terms.

Based on the interior regularity (interior understood as the set of $(x, t)$ where $v<<\epsilon$ ) in the parabolic operator, the following definition is provided:

Definition 2. Given the pairs of functions $(\hat{u}, \hat{v})$ and $(\tilde{u}, \tilde{v}) \in C^{2+\gamma, 1+\gamma / 2}\left(\mathbb{R}^{d} \times(0, T)\right)$. The pair $(\hat{u}, \hat{v})$ is formed of upper solutions if:

$$
L \hat{u} \geq \hat{v}^{n}, L \hat{v} \geq-\tilde{u}^{m} .
$$


The pair $(\tilde{u}, \tilde{v})$ is formed of lower solutions if:

$$
L \tilde{u} \leq \tilde{v}^{n}, L \tilde{v} \leq-\hat{u}^{m} .
$$

Note that:

$$
\tilde{u}(x, 0) \leq u(x, 0) \leq \hat{u}(x, 0), \quad \tilde{v}(x, 0) \leq v(x, 0) \leq \hat{v}(x, 0),
$$

where

$$
\hat{u}(x, 0)=u(x, 0)+\nu, \hat{v}(x, 0)=v(x, 0)+\nu, \tilde{u}(x, 0)=u(x, 0)-\nu, \tilde{v}(x, 0)=v(x, 0)-\nu .
$$

being

$$
0<\nu<\min _{x \in \mathbb{R}^{d}}(u(x, 0), v(x, 0)),
$$

Under the assumption that the absorption and reaction terms are Hölder continuous and that $u^{n}>0, v^{p}>0$ in a inner region with $v<<\epsilon$, the regularity of the parabolic operator (see [21] and Ch. $3[30])$ permits to state preliminary that the upper and lower solutions $(\hat{u}, \hat{v})$ and $(\tilde{u}, \tilde{v})$ $\in L^{1}\left(\mathbb{R}^{d} \times(0, T)\right)$.

Now, let define the monotone sequences referred as $\left(u^{(k)}, v^{(k)}\right)_{k=0,1,2, \ldots}$ with initial data $\left(u^{(0)}, v^{(0)}\right)$ :

$$
\begin{gathered}
u_{t}^{(k)}=\Delta\left(u^{(k)}\right)^{m}+c \cdot \nabla u^{(k)}+\left(v^{(k-1)}\right)^{n}, \\
v_{t}^{(k)}=\Delta\left(v^{(k)}\right)^{m}+c \cdot \nabla v^{(k)}-\left(u^{(k-1)}\right)^{p}, \\
u^{(k)}(x, 0)=u_{0}(x), v^{(k)}(x, 0)=v_{0}(x), \\
k=1,2,3 \ldots
\end{gathered}
$$

The notation is simplified as follows:

$$
\begin{gathered}
\left(u^{(k)}\right)^{m}=u^{(k) m}, \quad\left(v^{(k)}\right)^{m}=v^{(k) m},\left(v^{(k-1)}\right)^{n}=v^{(k-1) n},\left(u^{(k-1)}\right)^{p}=u^{(k-1) p}, \\
k=1,2,3 \ldots
\end{gathered}
$$

And shall be understood as the $k$ or $k-1$ sequence component to $m, n$ or $p$ respectively.

The system is now uncoupled with Lipschitz reaction and absorption. Then, there exist solutions $\left(u^{(k)}, v^{(k)}\right)$ based on the regularity of the parabolic operator $L$ (Ch. 3 [30] and analogous to Theorems 2.1 and 2.2, Ch. 7 [25]).

The monotone and ordered properties of the sequence $\left(u^{(k)}, v^{(k)}\right)$ can be proved following the approach in Ch. 12 of [25]. Given the mixed monotone forcing terms $\left(v^{n},-u^{p}\right)$, the sequence of upper solutions $\left(\hat{u}^{(k)}, \hat{v}^{(k)}\right)_{k=0,1,2, \ldots}$ and of lower solutions $\left(\tilde{u}^{(k)}, \tilde{v}^{(k)}\right)_{k=0,1,2, \ldots}$ with initial data (2.31) are monotone:

$$
\tilde{u} \leq \tilde{u}^{(k+1)} \leq \hat{u}^{(k+1)} \leq \tilde{u} ; \quad \tilde{v} \leq \tilde{v}^{(k+1)} \leq \hat{v}^{(k+1)} \leq \tilde{v},
$$

and ordered:

$$
\hat{u}^{(k)} \geq \hat{u}^{(k+1)} ; \quad \tilde{u}^{(k)} \leq \tilde{u}^{(k+1)} ; \quad \hat{v}^{(k)} \geq \hat{v}^{(k+1)} ; \quad \tilde{v}^{(k)} \leq \tilde{v}^{(k+1)} .
$$

The sequence of the upper solutions $\left(\hat{u}^{(k)}, \hat{v}^{(k)}\right)$ is non-increasing while the sequence of lower solutions $\left(\tilde{u}^{(k)}, \tilde{v}^{(k)}\right)$ is non-decreasing. Based on the monotone and ordered properties shown, the following existence and uniqueness theorem holds

Theorem 2. Let assume $(u, v)>(0,0)$ in $\mathbb{R}^{d} \times(0, T)$, then the defined sequences $\left(\hat{u}^{(k)}, \hat{v}^{(k)}\right)$ and $\left(\tilde{u}^{(k)}, \tilde{v}^{(k)}\right) \in C^{2+\gamma, 1+\gamma / 2}\left(\mathbb{R}^{d} \times(0, T)\right)$ are convergent.

Proof. Let consider the test function $\phi \in C^{\infty}\left(\mathbb{R}^{d}\right)$, such that for $0<\tau<t<T$, the upper and lower solutions $\hat{u}^{(k)}$ and $\tilde{u}^{(k)}$ can be constructed as:

$$
\begin{aligned}
& \int_{\mathbb{R}^{d}} \hat{u}^{(k)}(t) \phi(t)=\int_{\mathbb{R}^{d}}\left(u_{0}+\nu(k)\right) \phi(0)+\int_{0}^{t} \int_{\mathbb{R}^{d}}\left[\hat{u}^{(k)} \phi_{t}+\hat{u}^{(k) m} \Delta \phi+c \cdot \nabla \phi \hat{u}^{(k)}+\hat{v}^{(k) n} \phi\right] d s, \\
& \int_{\mathbb{R}^{d}} \tilde{u}^{(k)}(t) \phi(t)=\int_{\mathbb{R}^{d}}\left(u_{0}-\nu(k)\right) \phi(0)+\int_{0}^{t} \int_{\mathbb{R}^{d}}\left[\tilde{u}^{(k)} \phi_{t}+\tilde{u}^{(k) m} \Delta \phi+c \cdot \nabla \phi \tilde{u}^{(k)}+\tilde{v}^{(k) n} \phi\right] d s,
\end{aligned}
$$


where $\lim _{k \rightarrow \infty} \nu=0$. As per the dominated-convergence theorem:

$$
\begin{aligned}
\int_{\mathbb{R}^{d}} \lim _{k \rightarrow \infty} \hat{u}^{(k)}(t) \phi(t) & =\int_{\mathbb{R}^{d}}\left(u_{0}+\lim _{k \rightarrow \infty} \nu(k)\right) \phi(0) \\
& +\int_{0}^{t} \int_{\mathbb{R}^{d}}\left[\lim _{k \rightarrow \infty} \hat{u}^{(k)} \phi_{t}+\lim _{k \rightarrow \infty} \hat{u}^{(k) m} \Delta \phi+c \cdot \nabla \phi \lim _{k \rightarrow \infty} \hat{u}^{(k)}+\lim _{k \rightarrow \infty} \hat{v}^{(k) n} \phi\right] d s, \\
\int_{\mathbb{R}^{d}} \lim _{k \rightarrow \infty} \tilde{u}^{(k)}(t) \phi(t) & =\int_{\mathbb{R}^{d}}\left(u_{0}-\lim _{k \rightarrow \infty} \nu(k)\right) \phi(0) \\
& +\int_{0}^{t} \int_{\mathbb{R}^{d}}\left[\lim _{k \rightarrow \infty} \tilde{u}^{(k)} \phi_{t}+\lim _{k \rightarrow \infty} \tilde{u}^{(k) m} \Delta \phi+c \cdot \nabla \phi \lim _{k \rightarrow \infty} \tilde{u}^{(k)}+\lim _{k \rightarrow \infty} \tilde{v}^{(k) n} \phi\right] d s,
\end{aligned}
$$

and making the subtraction:

$$
\begin{aligned}
\int_{\mathbb{R}^{d}} \lim _{k \rightarrow \infty}\left(\hat{u}^{(k)}-\tilde{u}^{(k)}\right)(t) \phi(t) & =\int_{0}^{t} \int_{\mathbb{R}^{d}}\left[\lim _{k \rightarrow \infty}\left(\hat{u}^{(k)}-\tilde{u}^{(k)}\right) \phi_{t}+\lim _{k \rightarrow \infty}\left(\hat{u}^{(k) m}-\tilde{u}^{(k) m}\right) \Delta \phi\right. \\
& \left.+c \cdot \nabla \phi \lim _{k \rightarrow \infty}\left(\hat{u}^{(k)}-\tilde{u}^{(k)}\right)+\lim _{k \rightarrow \infty}\left(\hat{v}^{(k) n}-\tilde{v}^{(k) n}\right) \phi\right] d s,
\end{aligned}
$$

In the assumption that $\hat{v}^{(k)} \rightarrow \tilde{v}^{(k)}$, the last equality holds provided that $\hat{u}^{(k)} \rightarrow \tilde{u}^{(k)}$. The same steps can be repeated for the solution $v$. In this case, under the assumption that $\hat{u}^{(k)} \rightarrow \tilde{u}^{(k)}$, get $\hat{v}^{(k)} \rightarrow \tilde{v}^{(k)}$, showing then, the correctness of the initial assumption.

Additionally, the regular convergence involved in the upper and lower positive solutions permits to state that such solutions satisfy the regularity embedding:

$$
C^{2+\gamma, 1+\gamma / 2}\left(\mathbb{R}^{d} \times(0, T)\right) \subset L^{1}\left((0, T) ; W^{2,1}\left(\mathbb{R}^{d}\right)\right) \cap W^{1,1}\left((0, T) ; L^{1}\left(\mathbb{R}^{d}\right)\right)
$$

Theorem 3. Let assume $(u, v)>(0,0)$ is a minimal solution for $P(1.4)$ in $\mathbb{R}^{d} \times(0, T)$, then $(u, v)$ coincides with the maximal solution, i.e., the solution is unique.

Proof. Let $(\hat{u}, \hat{v})$ be the maximal solution to $P$ in $\mathbb{R}^{d} \times(0, T)$ such that:

$$
(\hat{u}(x, 0), \hat{v}(x, 0))=\left(u_{0}(x)+\nu, v_{0}(x)+\nu\right),
$$

with $\nu>0$ arbitrary small, defined as:

$$
\begin{aligned}
& \hat{u}_{t}=\Delta \hat{u}^{m}+c \cdot \nabla \hat{u}+\hat{v}^{n}, \\
& \hat{v}_{t}=\Delta \hat{v}^{m}+c \cdot \nabla \hat{v}-u^{p},
\end{aligned}
$$

and the minimal solution defined as:

$$
(u(x, 0), v(x, 0))=\left(u_{0}(x), v_{0}(x)\right),
$$

such that:

$$
\begin{aligned}
& u_{t}=\Delta u^{m}+c \cdot \nabla u+v^{n}, \\
& v_{t}=\Delta v^{m}+c \cdot \nabla v-\hat{u}^{p} .
\end{aligned}
$$

Then for every test function $\phi \in C^{\infty}\left(\mathbb{R}^{d}\right)$, the following expressions hold:

$$
\begin{aligned}
& 0 \leq \int_{\mathbb{R}^{d}}(\hat{u}-u)(t) \phi(t)=\int_{0}^{t} \int_{\mathbb{R}^{d}}\left[(\hat{u}-u) \phi_{t}+\left(\hat{u}^{m}-u^{m}\right) \Delta \phi+c \cdot \nabla \phi(\hat{u}-u)+\left(\hat{v}^{n}-v^{n}\right) \phi\right] d s \\
& 0 \leq \int_{\mathbb{R}^{d}}(\hat{v}-v)(t) \phi(t)=\int_{0}^{t} \int_{\mathbb{R}^{d}}\left[(\hat{v}-v) \phi_{t}+\left(\hat{v}^{m}-v^{m}\right) \Delta \phi+c \cdot \nabla \phi(\hat{v}-v)-\left(\hat{u}^{p}-u^{p}\right) \phi\right] d s .
\end{aligned}
$$

Let define:

$$
a_{1}(x, s)=\left[\begin{array}{cc}
\frac{\hat{u}^{m}-u^{m}}{\hat{u}-u} & \text { for } \hat{u} \not \equiv u \\
m u^{m-1} & \text { otherwise }
\end{array}\right]
$$




$$
a_{2}(x, s)=\left[\begin{array}{ll}
\frac{\hat{v}^{m}-v^{m}}{\hat{v}-v} & \text { for } \hat{v} \not \equiv v \\
m v^{m-1} & \text { otherwise }
\end{array}\right] .
$$

Given two fixed values for $x$ and $s=t \leq T$ :

$$
\begin{aligned}
& 0 \leq a_{1}(x, s) \leq c_{1}\left(m,\left\|u_{0}\right\|_{\infty}, T\right), \\
& 0 \leq a_{2}(x, s) \leq c_{2}\left(m,\left\|v_{0}\right\|_{\infty}, T\right) .
\end{aligned}
$$

Consider the test function:

$$
\phi(|x|, s)=e^{k(T-s)}\left(1+|x|^{2}\right)^{-\gamma},
$$

for some constants $k$ and $\gamma$.

The test function verifies:

$$
\begin{gathered}
\phi_{t}=-k \phi(x, s), \\
\left|\nabla_{|x|} \phi\right| \leq c_{3}(\gamma, d) \phi(x, s), \\
\Delta_{|x|} \phi \leq c_{4}(\gamma, d) \phi(x, s),
\end{gathered}
$$

such that:

$$
\begin{aligned}
(\hat{u}-u) \phi_{t}+\left(\hat{u}^{m}-u^{m}\right) \Delta \phi & +c \cdot \nabla \phi(\hat{u}-u)+\left(\hat{v}^{n}-v^{n}\right) \phi \leq-(\hat{u}-u) k \phi \\
& +a_{1}(\hat{u}-u) c_{4} \phi+c c_{3} \phi(\hat{u}-u)+L_{1}(\hat{v}-v) \phi \\
(\hat{v}-v) \phi_{t}+\left(\hat{v}^{m}-v^{m}\right) \Delta \phi & +c \cdot \nabla \phi(\hat{v}-v)+\left(\hat{u}^{p}-u^{p}\right) \phi \leq-(\hat{v}-v) k \phi \\
& +a_{1}(\hat{v}-v) c_{4} \phi+c c_{3} \phi(\hat{v}-v)+L_{2}(\hat{u}-u) \phi .
\end{aligned}
$$

where $L_{1}$ and $L_{2}$ refer to the Lipschitz constants.

The constant $k$ shall be selected as:

$$
-k+a_{1} c_{4}+c_{3} c \leq 0, \quad-k+a_{2} c_{4}+c_{3} c \leq 0 .
$$

It suffices to consider:

$$
k \geq \max \left(a_{1} c_{4}+c_{3} c, a_{2} c_{4}+c_{3} c\right),
$$

so that:

$$
\begin{aligned}
& \left(-k+a_{1} c_{4}+c_{3} c\right) \phi(\hat{u}-u) \leq 0, \\
& \left(-k+a_{1} c_{4}+c_{3} c\right) \phi(\hat{v}-v) \leq 0 .
\end{aligned}
$$

The expressions (2.47) and (2.48) read:

$$
\begin{aligned}
& 0 \leq \int_{\mathbb{R}^{d}}(\hat{u}-u)(t) \phi(t) \leq \int_{0}^{t} \int_{\mathbb{R}^{d}} L_{1}(\hat{v}-v) \phi d s, \\
& 0 \leq \int_{\mathbb{R}^{d}}(\hat{v}-v)(t) \phi(t) \leq \int_{0}^{t} \int_{\mathbb{R}^{d}} L_{2}(\hat{u}-u) \phi d s .
\end{aligned}
$$

Making the $d / d t$ in the previous expressions:

$$
\begin{aligned}
& 0 \leq \frac{d}{d t} \int_{\mathbb{R}^{d}}(\hat{u}-u)(t) \phi(t) \leq \int_{\mathbb{R}^{d}} L_{1}(\hat{v}-v)(t) \phi(t), \\
& 0 \leq \frac{d}{d t} \int_{\mathbb{R}^{d}}(\hat{v}-v)(t) \phi(t) \leq \int_{\mathbb{R}^{d}} L_{2}(\hat{u}-u)(t) \phi(t),
\end{aligned}
$$

Let operate in the last two expressions with the equality, then:

$$
\int_{\mathbb{R}^{d}}(\hat{u}-u)(t) \phi(t)=\frac{1}{L_{2}} \frac{d}{d t} \int_{\mathbb{R}^{d}}(\hat{v}-v)(t) \phi(t),
$$


which can be replaced in (2.62):

$$
\frac{d^{2}}{d t^{2}} \int_{\mathbb{R}^{d}}(\hat{v}-v)(t) \phi(t) \leq L_{1} L_{2} \int_{\mathbb{R}^{d}}(\hat{v}-v)(t) \phi(t) .
$$

Let define:

$$
g(t)=\int_{\mathbb{R}^{d}}(\hat{v}-v)(t) \phi(t),
$$

then $(2.65)$ reads as a linear standard second order equation:

$$
\frac{d^{2} g(t)}{d t^{2}}=L_{1} L_{2} g(t)
$$

with

$$
\begin{aligned}
& g(0)=\nu \rightarrow 0, \\
& g \prime(0)=\nu \rightarrow 0 .
\end{aligned}
$$

Then the equation (2.67) converges to the null solution. For the shake of simplicity consider $g(t)=0$, concluding that:

$$
\hat{v}=v
$$

Now, returning to $(2.60)$ :

$$
0 \leq \int_{\mathbb{R}^{d}}(\hat{u}-u)(t) \phi(t) \leq 0,
$$

to conclude:

$$
\hat{u}=u,
$$

showing, then, the uniqueness of any positive solution to $P$.

The decreasing rate of $v$ down to the proximity of the null solution leads to consider the loss of the Lipschitz condition. As a consequence, the following truncate for $v^{n}$ is introduced :

$$
v^{n}=\left[\begin{array}{c}
\epsilon^{n-1} v \text { for } 0 \leq v<\epsilon \\
v^{n} \text { for } v \geq \epsilon
\end{array}\right],
$$

which is used to approximate the non-Lipschitz problem by a Lipschitz one. Note that in the limit $\epsilon \rightarrow 0$, the original non-Lipschitz term is recovered. Therefore, $L_{1}=\epsilon^{n-1}$, so that the expression $(2.67)$ reads:

$$
\frac{d^{2} g(t)}{d t^{2}}=\epsilon^{n-1} L_{2} g(t),
$$

with the initial conditions given in (2.68). For an arbitrary small $\epsilon$ the solution to $g(t)$ is kept null. Then, uniqueness for the positive $u$ is kept when $v$ approaches the lower threshold $\epsilon$ (see the form of solutions given in $(2.27)$ ).

\section{Comparison of solutions}

This section follows a similar approach to that already contemplated in [15]. Some of the concepts are reproduced here for completeness. Given a solution $u_{2}>u_{1}$, the absorption term in the equation in $v$ would lead to have $v_{2}<v_{1}$ that, in turn, will lead to a lower magnitude in $u$. Therefore, $u$ would be lower in the next step, providing a lower absorption in the equation for $v$, that will result in a higher value for $v$. Again, this higher value for $v$ provides a higher value for $u$. This oscillatory dynamic introduces difficulties when arising a comparison principle that can be, only, formulated locally.

Let introduce a controlling parameter, $\kappa$, to establish certain conditions in which comparison holds. The following problem, referred as $P_{\kappa}$, is defined accordingly:

$$
\begin{aligned}
u_{t} & =\Delta u^{m}+c \cdot \nabla u+\kappa v^{n}, \\
v_{t} & =\Delta v^{m}+c \cdot \nabla v-\kappa u^{p} .
\end{aligned}
$$


Theorem 4. Given two pairs of positive solutions such that

$$
\left(u_{1}, v_{1}\right),\left(u_{2}, v_{2}\right) \in C^{2+\gamma, 1+\gamma / 2}\left(\mathbb{R}^{d} \times(0, T)\right),
$$

understood in each component wise for the problem $P_{\kappa}$ and that

$$
\begin{aligned}
& u_{0,1} \geq u_{0,2}>0 \in L^{1}\left(\mathbb{R}^{d}\right) \cap L^{\infty}\left(\mathbb{R}^{d}\right), \\
& v_{0,1} \geq v_{0,2}>0 \in L^{1}\left(\mathbb{R}^{d}\right) \cap L^{\infty}\left(\mathbb{R}^{d}\right),
\end{aligned}
$$

then:

$$
u_{1} \geq u_{2} ; \quad v_{1} \geq v_{2}
$$

locally, for all $(x, t) \in \beta\left(x_{0}, t\right) \times(0, T)$, where:

$$
\beta\left(x_{0}, t\right)=\left[x \in\left(x_{0}-B(t), x_{0}+B(t)\right) ; x_{0} \in \mathbb{R}^{d}\right] .
$$

And $T$ is sufficiently small (i.e. $T \rightarrow 0$ ).

Proof. The proof follows the same steps as in [15]. It suffices to replace $u, v$ by $u^{m}, v^{m}$ in the diffusion. Let define a region $\gamma\left(x_{0}, t\right)$ in the vicinity of $x_{0}$ and contained within the set $\beta\left(x_{0}, t\right)$, so that the solutions order is the opposite compared to the results to prove:

$$
\gamma\left(x_{0}, t\right)=\left[x \in\left(x_{0}-\sigma(t), x_{0}+\sigma(t)\right) ; x_{0} \in \mathbb{R}^{d} ; u_{2}>u_{1}, v_{2}>v_{1}\right],
$$

with

$$
\gamma\left(x_{0}, t\right) \subset \beta\left(x_{0}, t\right) .
$$

In the border of $\gamma, \partial \gamma\left(x_{0}, t\right)$ :

$$
u_{1}=u_{2} ; v_{1}=v_{2} \text {. }
$$

The postulated solutions $u_{1}, u_{2}, v_{1}, v_{2}$ satisfy the problem $P_{\kappa}$. Making the subtraction, arranging the terms in vectorial form and integrating over $\gamma\left(x_{0}, t\right)$ :

$$
\begin{aligned}
& \frac{d}{d t} \int_{\gamma(t)}\left(\begin{array}{c}
u_{2}-u_{1} \\
v_{2}-v_{1}
\end{array}\right) d x=\int_{\gamma(t)}\left(\begin{array}{c}
\Delta\left(u_{2}^{m}-u_{1}^{m}\right) \\
\Delta\left(v_{2}^{m}-v_{1}^{m}\right)
\end{array}\right) d x \\
& \quad+\int_{\gamma(t)}\left(\begin{array}{c}
c \cdot \nabla\left(u_{2}-u_{1}\right) \\
c \cdot \nabla\left(v_{2}-v_{1}\right)
\end{array}\right) d x+\int_{\gamma(t)} \kappa\left(\begin{array}{c}
v_{2}^{n}-v_{1}^{n} \\
-u_{2}^{p}+u_{1}^{p}
\end{array}\right) d x .
\end{aligned}
$$

Making use of the Green's formula:

$$
\int_{\gamma(t)}\left(\begin{array}{c}
\Delta\left(u_{2}^{m}-u_{1}^{m}\right) \\
\Delta\left(v_{2}^{m}-v_{1}^{m}\right)
\end{array}\right) d x=\int_{\partial \gamma(t)}\left(\begin{array}{c}
\nabla\left(u_{2}^{m}-u_{1}^{m}\right) \cdot \Pi \\
\nabla\left(v_{2}^{m}-v_{1}^{m}\right) \cdot \Pi
\end{array}\right) d x .
$$

Where $\Pi$ is the outer normal unit vector in $\partial \gamma(t)$. In the proximity of $\partial \gamma(t)$ :

$$
\begin{aligned}
& \nabla\left(u_{2}^{m}-u_{1}^{m}\right) \leq 0, \\
& \nabla\left(v_{2}^{m}-v_{1}^{m}\right) \leq 0 .
\end{aligned}
$$

Therefore:

$$
\int_{\partial \gamma(t)}\left(\begin{array}{c}
\nabla\left(u_{2}^{m}-u_{1}^{m}\right) \cdot \Pi \\
\nabla\left(v_{2}^{m}-v_{1}^{m}\right) \cdot \Pi
\end{array}\right) \leq\left(\begin{array}{l}
0 \\
0
\end{array}\right) .
$$

Note that the integral assessments in the advection and independent terms are omitted (see further details in [15]). After compilation:

$$
\frac{d}{d t} \int_{\gamma(t)}\left(\begin{array}{c}
u_{2}-u_{1} \\
v_{2}-v_{1}
\end{array}\right) d x \leq \int_{\gamma(t)} \kappa\left(\begin{array}{c}
\frac{n}{v_{1}^{1-n}}\left|v_{2}-v_{1}\right| \\
\frac{p}{u_{1}^{1-p}}\left|-u_{2}+u_{1}\right|
\end{array}\right) d x
$$

Note that a comparison principle does not hold in case the solutions $v$ or $u$ are equal to zero due to the lack of regularity. Nonetheless, if the initial conditions are positive, the Lipschitz condition (2.42) and the positivity of the lower solutions (2.31) hold, leading to positivity in the solutions. 
Now, the following Lipschitz constant is defined:

$$
K=\max \{p, n\} \frac{1}{\min _{\gamma(t)}\left\{v_{1}^{1-n}, u_{1}^{1-p}\right\}} .
$$

Returning to the integral assessment and after application of the Gronwall's inequality:

$$
\begin{aligned}
\frac{d}{d t} \int_{\gamma(t)}\left(\begin{array}{c}
u_{2}-u_{1} \\
v_{2}-v_{1}
\end{array}\right) d x & \leq \kappa K \int_{\gamma(t)}\left(\begin{array}{c}
\left|v_{0,2}-v_{0,1}\right| \\
\left|-u_{0,2}+u_{0,1}\right|
\end{array}\right) d x+ \\
& \kappa K \int_{\gamma(t)} \int_{0}^{t}\left(\begin{array}{c}
\left|v_{2}-v_{1}\right| \\
\left|-u_{2}+u_{1}\right|
\end{array}\right) d s d x .
\end{aligned}
$$

Consider $\sigma(t) \rightarrow 0$ so that for any time $B(t)>>\sigma(t)$ and the domain $\gamma(t)$ approximates the center point $x_{0}$ :

$$
\frac{d}{d t} \int_{\beta(t)}\left(\begin{array}{c}
u_{2}-u_{1} \\
v_{2}-v_{1}
\end{array}\right) d x \leq \frac{d}{d t} \int_{\gamma(t)}\left(\begin{array}{c}
u_{2}-u_{1} \\
v_{2}-v_{1}
\end{array}\right) d x .
$$

It is clear that in case of having $\kappa \rightarrow 0$ in the domain $\beta(t)$, the comparison principle applies, indeed:

$$
\frac{d}{d t} \int_{\beta(t)}\left(\begin{array}{c}
u_{2}-u_{1} \\
v_{2}-v_{1}
\end{array}\right) d x \leq\left(\begin{array}{c}
0 \\
0
\end{array}\right) \rightarrow\left(\begin{array}{c}
u_{2 t} \leq u_{1 t} \\
v_{2 t} \leq v_{1 t}
\end{array}\right)_{\beta},
$$

which means that

$$
\left(\begin{array}{l}
d u_{2} \leq d u_{1} \\
d v_{2} \leq d v_{1}
\end{array}\right)_{\beta}
$$

This last expression can be evaluated as:

$$
\left(\begin{array}{c}
u_{2}-u_{2}(\partial \gamma) \leq u_{1}-u_{1}(\partial \gamma) \\
v_{2}-v_{2}(\partial \gamma) \leq v_{1}-v_{1}(\partial \gamma)
\end{array}\right)_{\beta}
$$

where $u_{i}(\partial \gamma)$ and $v_{i}(\partial \gamma)$ represent the evaluation of each function in the border of the domain $\gamma$. Note that the expression (3.8) holds, so:

$$
\left(\begin{array}{l}
u_{2} \leq u_{1} \\
v_{2} \leq v_{1}
\end{array}\right)_{\beta}
$$

as it was the intention to proof. The index $\beta(t)$ stresses the fact that the comparison holds locally as described in the theorem postulations.

Nonetheless, if $\kappa>a>0$ (for example $\kappa=1$ ), a comparison holds provided the initial conditions are sufficiently close and that $t=T \rightarrow 0$. Under these assumptions:

$$
\begin{aligned}
& u_{2} \leq u_{1}, \\
& v_{2} \leq v_{1}
\end{aligned}
$$

Locally in $\beta\left(x_{0}, t\right)$.

In addition, the following lemma enunciates:

Lemma 1. Let consider

$$
u_{0} \geq v_{0}>0
$$

for $x \in \mathbb{R}^{d}$, then

$$
u \geq v>0
$$

for any $(x, t) \in \mathbb{R}^{d} \times(0, T)$. 
Proof. Any pair of solutions $(u, v)$ satisfies the equations (2.1) and (2.2). Subtracting both:

$\int_{\mathbb{R}^{d}}(u-v)(t) \phi(t)=\int_{\mathbb{R}^{d}}\left(u_{0}-v_{0}\right) \phi(0)+\int_{0}^{t} \int_{\mathbb{R}^{d}}\left[(u-v) \phi_{t}+\left(u^{m}-v^{m}\right) \Delta \phi+c \cdot \nabla \phi(u-v)+\left(v^{n}+u^{p}\right) \phi\right] d s$,

so that:

$$
\begin{aligned}
\int_{\mathbb{R}^{d}}(u-v)(t) \phi(t) & -\int_{0}^{t} \int_{\mathbb{R}^{d}}\left[(u-v) \phi_{t}+\left(u^{m}-v^{m}\right) \Delta \phi+c \cdot \nabla \phi(u-v)\right. \\
& =\int_{\mathbb{R}^{d}}\left(u_{0}-v_{0}\right) \phi(0)+\int_{0}^{t} \int_{\mathbb{R}^{d}}\left(v^{n}+u^{p}\right) \phi d s \geq 0 .
\end{aligned}
$$

where the test function $\phi$ is given in (2.53), so that in the asymptotic condition for $|x|>>1$ :

$$
\phi_{t}=-k \phi, \quad \phi_{x} \sim-2|x|^{-1} \gamma \phi, \quad \phi_{x x} \sim 2|x|^{-2} \gamma \phi .
$$

Consider from (3.25) :

$$
\int_{\mathbb{R}^{d}}(u-v)(t) \phi(t)-\int_{0}^{t} \int_{\mathbb{R}^{d}}\left[(u-v) \phi_{t}+\left(u^{m}-v^{m}\right) \Delta \phi+c \cdot \nabla \phi(u-v) \geq 0 .\right.
$$

After application of the Gronwall's inequality:

$$
\begin{aligned}
\int_{\mathbb{R}^{d}}(u-v) \phi & +\int_{\mathbb{R}^{d}} K_{1}(u-v) k \phi-\int_{\mathbb{R}^{d}} K_{2}\left(u_{0}-v_{0}\right) k \phi-\int_{\mathbb{R}^{d}} K_{3}\left(u^{m}-v^{m}\right) \frac{2}{|x|^{2}} \gamma \phi \\
& +\int_{\mathbb{R}^{d}} K_{4}\left(u_{0}^{m}-v_{0}^{m}\right) \frac{2}{|x|^{2}} \gamma \phi+\int_{\mathbb{R}^{d}} K_{5}(u-v) \frac{2 c}{|x|} \gamma \phi-\int_{\mathbb{R}^{d}} K_{6}\left(u_{0}-v_{0}\right) \frac{2 c}{|x|} \gamma \phi \geq 0
\end{aligned}
$$

where $K_{i}>0$ for $i=\{1,2,3,4,5,6\}$ represent the set of constant as per the Gronwall's inequality. In the infinitesimal approach:

$$
\int_{\mathbb{R}^{d}}(u-v) \phi+\int_{\mathbb{R}^{d}} K_{1}(u-v) k \phi \geq \int_{\mathbb{R}^{d}} K_{2}\left(u_{0}-v_{0}\right) k \phi \geq 0,
$$

so that:

$$
\int_{\mathbb{R}^{d}}(u-v) \phi+\int_{\mathbb{R}^{d}} K_{1}(u-v) k \phi \geq 0
$$

Given the positivity of the test functions, this last condition holds provided that

$$
u \geq v
$$

\section{Profiles of solution}

This section is devoted to the searching of upper and lower solutions considering that the invasive specie $u(x, t)$ shall be non-decreasing (i.e. $u_{t} \geq 0$ ) while the invaded one $v(x, t)$ shall be nonincreasing (i.e. $v_{t} \leq 0$ ).

Theorem 5. The maximal solution $\hat{v}(x, t)$ for the invaded specie is:

$$
\hat{v}(x, t)=v_{0}(x)-c_{v}(m, d, p) t^{\frac{1}{m-1}},
$$

where

$$
c_{v}(m, d, p)=\left(\frac{d(m-1)-1}{m-1}\right)^{\frac{1}{p-1}}
$$

for

$$
0<t<\frac{|x|}{c}
$$


In addition, $\hat{v}$ propagates along the support in $(x, t)$ given by:

$$
|x|=c_{s}(d, m, p) t
$$

where

$$
c_{s}=\left(\frac{\left(d-\frac{1}{m-1}\right)^{\frac{m-1}{p-1}}}{\frac{m-1}{2 m}}\right)^{\frac{1}{2}} .
$$

Proof. Let start with the equation in $v$ :

$$
v_{t}=\Delta v^{m}+c \cdot \nabla v-u^{p},
$$

considering the Lipschitz approximation for $u^{p}$ and the comparison shown in Lemma 1:

$$
g_{\epsilon}=\min \left\{u^{p}, \epsilon^{p-1} u\right\} \geq h_{\epsilon}=\min \left\{v^{p}, \epsilon^{p-1} v\right\} .
$$

In the search of selfsimilar solutions:

$$
\hat{v}(x, t)=t^{-\alpha} f(\xi), \quad \xi=|x| t^{\beta},
$$

to the upper profile equation:

$$
\hat{v}_{t}=\Delta \hat{v}^{m}+c \cdot \nabla \hat{v}-\hat{v}^{p}
$$

the following holds:

$$
-\alpha t^{-\alpha-1} f+\beta \xi t^{-\alpha-1} f^{\prime}=G\left(f^{\prime \prime}, f^{\prime}, f, m\right) t^{-\alpha m+2 \beta}+c t^{-\alpha+\beta} f^{\prime}-h_{\epsilon} .
$$

Making the corresponding equalities in the time exponents:

$$
\alpha=\frac{-1}{m-1} ; \quad \beta=-1 .
$$

The selfsimilar profile shall, then, be obtained as a solution to the elliptic equation:

$$
-\alpha f+\beta \xi f^{\prime}=\left(f^{m}\right)^{\prime \prime}+\frac{d-1}{\xi}\left(f^{m}\right)^{\prime}+c f^{\prime}-c_{1} f,
$$

such that

$$
h_{\epsilon} \geq c_{1} f,
$$

for a sufficiently large $t$ to be determined. Further, let consider:

$$
c_{1}=\alpha-\beta d=\alpha+d .
$$

Note that in the approximation:

$$
\beta \xi>>c, \quad|x| t^{-1}>>|-c|,
$$

the following elliptic equation holds for $t<<\frac{|x|}{c}$.

$$
\beta \xi f^{\prime}=\left(f^{m}\right)^{\prime \prime}+\frac{d-1}{\xi}\left(f^{m}\right)^{\prime}+\beta d f,
$$

for which a solution is available [30] and [12]:

$$
f(\xi)=\left(A-B \xi^{2}\right)^{\frac{1}{m-1}},
$$

where:

$$
\begin{gathered}
A>0, \\
B=\frac{(1-m) \beta}{2 m} .
\end{gathered}
$$


These last solution is valid provided:

$$
t_{1}<<t<<\frac{|x|}{c}
$$

where $t_{1}$ is obtained in the sub-linear approximation (4.7) upon replacement of the selfsimilar solution:

$$
\epsilon^{p-1} t^{(p-1)} f>c_{1} f
$$

then

$$
t>\left(\frac{\alpha+d}{\epsilon^{p-1}}\right)^{\frac{1}{\alpha(p-1)}}=c_{2}(\alpha, d, p) \epsilon^{-\alpha} .
$$

Admit:

$$
t_{1}=c_{2}(\alpha, d, p) \epsilon^{-\alpha}=c_{2}(\alpha, d, p) \epsilon^{\frac{1}{m-1}},
$$

so that:

$$
t_{1} \rightarrow 0
$$

whenever $\epsilon \rightarrow 0$ to recover the original non-Lipschitz term.

$A$ is obtained by making $\xi=0$ in (4.17), so that:

$$
\hat{v}(x, t)=A^{\frac{1}{m-1}} t^{-\alpha},
$$

then,

$$
\min \left\{f^{p}, \epsilon^{p-1} t^{\alpha(p-1)} f\right\} \geq(\alpha+d) f ; \min \left\{f^{p-1}, \epsilon^{p-1} t^{\alpha(p-1)}\right\} \geq(\alpha+d),
$$

Note that for $\xi=0$ and according to (4.17):

$$
\min \left\{A^{\frac{p-1}{m-1}}, \epsilon^{p-1} t^{\alpha(p-1)}\right\} \geq(\alpha+d),
$$

then

$$
A=(\alpha+d)^{\frac{m-1}{p-1}}>0 .
$$

The maximal time evolution $\hat{v}$ is then given by:

$$
\hat{v}(x, t)=v_{0}(x)-c_{v}(m, d, p) t^{\frac{1}{m-1}},
$$

where

$$
c_{v}(m, d, p)=\left(\frac{d(m-1)-1}{m-1}\right)^{\frac{1}{p-1}} .
$$

Finally, $\hat{v}$ is indeed an upper evolution. Note that $\hat{v}$ has been obtained with a lower Lipschitz approximation:

$$
u^{p}>h_{\epsilon}>v^{p}>g_{\epsilon} .
$$

Operating with $g_{\epsilon}$, instead of $u^{p}$, provides a regular condition to obtain the upper profile $\hat{v}$. To show this, let consider a solution $v$ to the original equation given by the absorption term $-u^{p}$ and define a $t_{0}$ :

$$
0<t_{0}<\frac{|x|}{c}
$$

such that for any $\tau$ with $t_{0}<\tau<\frac{|x|}{c}$ :

$$
v(x, \tau) \leq \hat{v}\left(x, t_{0}\right) .
$$

Now, for any $t$ with $\tau<t<\frac{|x|}{c}$ :

$$
v(x, \tau+t) \leq \hat{v}\left(x, t_{0}+t\right) .
$$

In the limit $\tau \rightarrow 0$ :

$$
v(x, t) \leq \hat{v}(x, t),
$$

showing the upper behaviour of $\hat{v}$ compared to $v$.

The propagating support is obtained making:

$$
f(\xi)=0 ; \quad A=B \xi^{2},
$$


so that $A$ and $B$ can be replaced by their corresponding values according to the expressions (4.27) and (4.18):

$$
|x|=c_{s}(d, m, p) t,
$$

where

$$
c_{s}=\left(\frac{\left(d-\frac{1}{m-1}\right)^{\frac{m-1}{p-1}}}{\frac{m-1}{2 m}}\right)^{\frac{1}{2}} .
$$

Note that solutions are of the form $(2.27)$ in $\mathbb{R}^{d} \times[0, \infty]$, so that

$$
\hat{v}=\max \{\hat{v}, \delta\}=A-c_{v} t^{\frac{1}{m-1}},
$$

in the proximity of $\delta \rightarrow 0^{+}$:

$$
\delta=A-c_{v} t^{\frac{1}{m-1}} .
$$

As $\delta<<A$ :

$$
t_{v}=\left(\frac{(\alpha+d)^{\frac{m-1}{p-1}}}{c_{v}}\right)^{m-1}
$$

The expression (4.40) provides an estimation in time to consider a vanishing condition for $v$, so that for $t>t_{v}$, the equation for $\tilde{u} \leq u$ reads:

$$
\tilde{u}_{t}=\Delta \tilde{u}^{m}+c \cdot \nabla \tilde{u},
$$

and the following theorem holds in the search of a lower solution $\tilde{u}$ :

Theorem 6. Let consider

$$
A_{1}=\max _{x \in \mathbb{R}^{d}}\left\{u_{0}(x)\right\} .
$$

The lower solution $\tilde{u}(x, t)$ for the invasive specie reads:

$$
\tilde{u}(x, t)=u_{0}(x)+A_{1} t^{\frac{1}{m-1}},
$$

for

$$
t_{v}<t<\frac{|x|}{c}
$$

Proof. Again, consider selfsimilar solutions of the form:

$$
\tilde{u}(x, t)=t^{-\alpha} F(\xi), \quad \xi=|x| t^{\beta},
$$

to the lower profile equation (4.41). Upon substitution of the selfsimilar solution (4.45) and operating analogously as in (4.10), the following selfsimilar exponents are determined:

$$
\alpha=\frac{-1}{m-1} ; \quad \beta=-1 .
$$

Assume $t<\frac{|x|}{c}$, the selfsimilar profile $F$ is obtained as a solution to the elliptic equation:

$$
\beta \xi F^{\prime}=\left(F^{m}\right)^{\prime \prime}+\frac{d-1}{\xi}\left(F^{m}\right)^{\prime}+\alpha f,
$$

for which the solution is, again, provided in [30] and [12]:

$$
F(\chi)=\left(A_{1}-B_{1} \xi^{2}\right)^{\frac{1}{m-1}}
$$

for $t_{v}<t<\frac{|x|}{c}$ and $A_{1}$ and $B_{1}$ as per the expressions (4.18).

Making $\xi=0$, the solution for $\tilde{u}$ is:

$$
\tilde{u}(x, t)=u_{0}(x)+A_{1} t^{\frac{1}{m-1}},
$$


where

$$
A_{1}=\max _{x \in \mathbb{R}^{d}}\left\{u_{0}(x)\right\} .
$$

Finally, the expression in (4.49) is shown to be a lower solution. For such purpose, let define $t_{0}$, such that $t_{v}<t_{0}<\frac{|x|}{c}$, and $\tau$ such that $t_{0}<\tau<\frac{|x|}{c}$. Let consider now:

$$
\tilde{u}(x, \tau) \leq u\left(x, t_{0}\right) .
$$

This last expression is valid provided $\left|t_{0}-\tau\right|<<1$. Let consider $t^{\prime}$ with $\tau<t^{\prime}<\frac{|x|}{c}$, then:

$$
\tilde{u}\left(x, \tau+t^{\prime}\right) \leq u\left(x, t_{0}+t^{\prime}\right) .
$$

After a simple time translation $t=t_{0}+t^{\prime}$ :

$$
\tilde{u}(x, t) \leq u(x, t),
$$

with $t_{v}<\frac{t}{2}<\frac{|x|}{c}$.

The next objective is to define a maximal solution for $u$ and a minimal solution for $v$. To this end, the following theorem holds:

Theorem 7. There exist a minimal solution $\tilde{v}(x, t)$ and a maximal solution $\hat{u}(x, t)$ in $\mathbb{R}^{d} \times[0, T]$ that adopt the form:

$$
\tilde{v}(x, t)=v_{0}(x)-K_{v}(n, p) t^{\frac{p+1}{1-n p}},
$$

where

$$
K_{v}(n, p)=\left(\left(\frac{p+1}{n+1}\right)^{\frac{p}{p+1}}\left(\frac{1-n p}{p+1}\right)\right)^{\frac{p+1}{1-n p}} .
$$

and

$$
\hat{u}(x, t)=u_{0}(x)+K_{u}(n, p) t^{\frac{n+1}{1-n p}},
$$

where

$$
K_{u}(n, p)=\left(\frac{p+1}{n+1}\right)^{\frac{1}{p+1}}\left(\left(\frac{p+1}{n+1}\right)^{\frac{p}{p+1}}\left(\frac{1-n p}{p+1}\right)\right)^{\frac{n+1}{1-n p}} .
$$

Proof. For building the maximal solution $\hat{u}(x, t)$, consider the following problem in $u$ :

$$
\begin{gathered}
u_{t}^{\epsilon}=\Delta\left(u^{\epsilon}\right)^{m}+c \cdot \nabla u^{\epsilon}+f_{\epsilon}(v), \\
u^{\epsilon}(x, 0)=u_{0}(x)+\epsilon, \quad v^{\epsilon}(x, 0)=v_{0}(x), \\
u_{0}(x)>0 . \quad v_{0}(x)>0 \in L^{1}\left(\mathbb{R}^{d}\right) \cap L^{\infty}\left(\mathbb{R}^{d}\right),
\end{gathered}
$$

where $f_{\epsilon}(v)$ is defined as per the expression (2.72). Solutions to problem (4.58) do exist under the Lipschitz condition in the reaction term and the positive initial conditions (refer to theorems 1 and 2).

Let consider $\epsilon_{2}<\epsilon$, so that:

$$
\begin{gathered}
u_{t}^{\epsilon}=\Delta\left(u^{\epsilon}\right)^{m}+c \cdot \nabla u^{\epsilon}+f_{\epsilon}(v), \\
u_{t}^{\epsilon}=\Delta\left(u^{\epsilon}\right)^{m}+c \cdot \nabla u^{\epsilon}+f_{\epsilon_{2}}(v), \\
u^{\epsilon}(x, 0)=u_{0}(x)+\epsilon>0 .
\end{gathered}
$$

The iteration process, starting with $\epsilon$, provides the condition $f_{\epsilon}(v)=f_{\epsilon_{2}}(v)$, then $u^{\epsilon_{2}}<u^{\epsilon}$ in accordance with the initial data for $u^{\epsilon}$ in (4.58). The same argument can be repeated for $\epsilon_{3}<\epsilon_{2}$, so that $u^{\epsilon_{3}}<u^{\epsilon_{2}}$. The sequence defined as $\left\{u^{\epsilon}\right\}$ is non-negative and non-increasing. Consequently the following maximal solution in the limit is defined:

$$
\hat{u}=\lim _{\epsilon \rightarrow 0} u^{\epsilon} .
$$

To construct the minimal solution for $v$, consider the problem:

$$
\begin{gathered}
v_{t}^{\epsilon}=\Delta\left(v^{\epsilon}\right)^{m}+c \cdot \nabla v^{\epsilon}-\left(\lim _{\epsilon \rightarrow 0} u^{\epsilon}\right)^{p}, \\
v(x, 0)=v_{0}(x)>0 .
\end{gathered}
$$


Solutions to (4.61) do exist (refer to theorems 1 and 2).

Consider $\epsilon_{2}<\epsilon$, then:

$$
u^{\epsilon_{2}}<u^{\epsilon}, \Rightarrow v^{\epsilon_{2}}>v^{\epsilon} .
$$

Therefore, the sequence $\left\{v^{\epsilon}\right\}$ is non-decreasing as $\epsilon \rightarrow 0$. The minimal solutions for $v$ is defined as per the monotone limit:

$$
\tilde{v}=\lim _{\epsilon \rightarrow 0} v^{\epsilon} .
$$

Once the maximal and minimal solutions have been constructed and shown to exist, the next intention is to search for a family of flat solutions $u^{\epsilon}, v^{\epsilon}$ via the resolution of:

$$
\begin{gathered}
u_{t}^{\epsilon}=\left(v^{\epsilon}\right)^{n}, \\
v_{t}^{\epsilon}=-\left(u^{\epsilon}\right)^{p}, \\
u^{\epsilon}(x, 0)=u_{0}(x)+\epsilon, \quad v^{\epsilon}(x, 0)=v_{0}(x), \\
u_{0}(x)>0 . \quad v_{0}(x)>0 \in L^{1}\left(\mathbb{R}^{d}\right) \cap L^{\infty}\left(\mathbb{R}^{d}\right) .
\end{gathered}
$$

This last system can be solved by separation of variables, so that:

$$
v^{\epsilon}(x, t)=v_{0}(x)-K_{v}(n, p) t^{\frac{p+1}{1-n p}},
$$

where

$$
K_{v}(n, p)=\left(\left(\frac{p+1}{n+1}\right)^{\frac{p}{p+1}}\left(\frac{1-n p}{p+1}\right)\right)^{\frac{p+1}{1-n p}}
$$

and

$$
u^{\epsilon}(x, t)=u_{0}(x)+\epsilon+K_{u}(n, p) t^{\frac{n+1}{1-n p}},
$$

where

$$
K_{u}(n, p)=\left(\frac{p+1}{n+1}\right)^{\frac{1}{p+1}}\left(\left(\frac{p+1}{n+1}\right)^{\frac{p}{p+1}}\left(\frac{1-n p}{p+1}\right)\right)^{\frac{n+1}{1-n p}} .
$$

Finally, $\hat{u}$ and $\tilde{v}$ are obtained in accordance with the expressions (4.60) and (4.63).

On a physical sound, note that each of the parameters $(d, m, c, n, p)$ involved in the minimal and maximal solutions shall be determined experimentally. Such an experimental process for a Porous Medium Equation has been followed in a mass transfer application for a fire extinguishing process [14]. In this cited reference the measured magnitude corresponded to the fire extinguisher concentration that shall be replaced by the specie concentration in the present analysis.

Depending on the particular application, either the maximal or minimal solution may be considered. For instance, if the intention is to model the minimal level of invaded specie that can feed an invasive to make it prosper, then the solution $\tilde{v}$ in (4.54) shall be taken into account together with the solution $\hat{u}$ in (4.56). In addition, note that the maximal solution for the invaded specie (and hence any other solution below) propagates along the support given in (4.4) which elucidates the finite propagation feature. Considering $d=2$, the support propagates along a circular domain of radium $\sqrt{c_{s} t}$, which makes sense in accordance with the non-linear diffusion. Note that within such propagating domain, the desertion will guide the invaded specie to move along the preferred direction $c$. If $c$ is high then the propagating support (4.4), induced by the diffusion, may only exist for a small time (see (4.3)). The short existence of such support reflects the fact that the invaded specie is living peacefully in the inner positive region $|x|<c_{s} t$, for $t$ according with (4.3), and that for $t \geq \frac{|x|}{c}$ the desertion starts as the invaded specie notes the invasive.

The positivity condition for $u$ permits to conclude on some regularity results in the quasilinear parabolic operator (see Section 2). On top, the specie $v$ verifies

$$
v \rightarrow \epsilon \rightarrow 0^{+} \quad \text { in } \quad B_{R}^{T}=B_{R}\left(x_{0} \cdot R\right) \times[T-\epsilon, T+\epsilon],
$$

which means the existence of a convergent tail in $B_{R}^{T}$ towards the null condition in $v$. The next objective is, then, to characterize the behaviour of $v$ and to conclude on a criteria to show the existence of finite propagation. 
Theorem 8. Any upper or lower solution for $v$ exhibits an exponential decreasing tail along the characteristic curve:

$$
y(x, t)=K \frac{m-1}{m}\left(t+\frac{x}{c}\right) .
$$

in the inner region

$$
|x|<<\frac{c}{m-2} t
$$

In addition and for $m>2$, there exists finite propagation speed when

$$
v \rightarrow \epsilon \rightarrow 0^{+} \quad \text { in } \quad B_{R}^{T}=B_{R}\left(x_{0} \cdot R\right) \times[T-\epsilon, T+\epsilon],
$$

where finite propagation refers to the existence of a positive convergent tail for $v$ approaching the null solution.

Proof. Consider the pressure variable $w$ :

$$
w=\frac{m}{m-1} v^{m-1},
$$

so that the equation $v$ in $P$ reads:

$$
\frac{m}{m-1} w_{t}=|\nabla w|^{2}+w \Delta w+c \cdot \nabla w \frac{m}{(m-1)^{2}}-(\delta+w)^{p} w^{\frac{m-2}{m-1}}
$$

where

$$
u=\delta+w, \quad \delta>0
$$

and $\delta$ almost constant. Assuming that $w \rightarrow 0$ :

$$
\frac{m}{m-1} w_{t}=|\nabla w|^{2}+c \cdot \nabla w \frac{m}{(m-1)^{2}}-\delta^{p} w^{\frac{m-2}{m-1}} .
$$

Let assume the asymptotic separation of variables:

$$
w(x, t)=g(t) \psi(x)+h(t) \gamma(x)+\ldots \text { where }|h(t)|<<|g(t)| \text {, as } t \rightarrow \infty \text {. }
$$

Replacing into (4.76) and considering the leading terms:

$$
\frac{m}{m-1} g^{\prime} \psi=\left(c \psi^{\prime} \frac{m}{m-1}-\delta^{p} \frac{1}{g^{\frac{1}{m-1}}} \psi^{\frac{m-2}{m-1}}\right) g,
$$

so that:

$$
\frac{m}{m-1} \frac{g^{\prime}}{g}=\frac{c \psi^{\prime} \frac{m}{m-1}-\delta^{p} \frac{1}{g^{\frac{1}{m-1}}} \psi^{\frac{m-2}{m-1}}}{\psi}=-K
$$

with $K>0$, then:

$$
g(t) \sim e^{-K \frac{m-1}{m} t} .
$$

For the resolution of $\psi$, consider that the following order is met under certain conditions to be specified later:

$$
\psi^{\frac{m-2}{m-1}}<<g^{\frac{1}{m-1}}=e^{-K \frac{1}{m} t} .
$$

Returning into (4.79), $\psi$ is obtained as:

$$
\psi(x)=e^{-K \frac{m-1}{m} \frac{1}{c} x}
$$

This solution is valid in the inner region given by:

$$
\psi^{\frac{m-2}{m-1}}<<e^{-K \frac{1}{m} t}, \quad|x|<<\frac{c}{m-2} t .
$$

Then, the pressure term adopts the solution:

$$
w(x, t) \sim e^{-K \frac{m-1}{m}\left(t+\frac{x}{c}\right)},
$$


where the characteristic curve is given by:

$$
y(x, t)=K \frac{m-1}{m}\left(t+\frac{x}{c}\right) .
$$

Observe the existence of an exponential profile for $w \rightarrow 0$ and, hence, for $v \rightarrow \epsilon \rightarrow 0^{+}$. When $m>2$ and $w \rightarrow 0$ the following expression holds:

$$
\frac{m}{m-1} w_{t}=|\nabla w|^{2}+c \cdot \nabla w \frac{m}{(m-1)^{2}} .
$$

A solution to a similar equation has been provided in [12]. Consider the following function in the search of a maximal solution:

$$
W(x, t)=a\left(b t+r-\frac{1}{n}\right)_{+}, r=|x|, n \in \mathbb{N} .
$$

Both $a$ and $b>0$ are constants to be determined. In particular, for $0 \leq \tau \leq 1$, impose:

$$
b \tau=\frac{1}{2 n} .
$$

Under this condition:

$$
W(x, t) \equiv 0 \quad \text { for } \quad r<\frac{1}{2 n} \text { and } 0 \leq t \leq \tau .
$$

Any solution to the equation (4.86) is bounded as per Theorem 1, then:

$$
v(x, t) \leq K_{1} \text { for } x \in \mathbb{R}, 0 \leq t \leq \tau \text { and } K_{1}\left(p,\left\|u_{0}\right\|_{\infty}\right) .
$$

The intention is to make $W(x, t)$ as a maximal solution:

$$
W(x, t) \geq v(x, t),
$$

so that

$$
a\left(b t+r-\frac{1}{n}\right)_{+} \geq K_{1} .
$$

Select any $r>\frac{1}{n}$, for example $r=\frac{2}{n}$. Thus, for $t=0$ :

$$
a\left(\frac{2}{n}-\frac{1}{n}\right)_{+} \geq K_{1}, \quad a \geq n K_{1} .
$$

Note that:

$$
W(x, t) \geq v(x, t),
$$

in $r=\frac{2}{n}$ and $0 \leq t \leq \tau$. The value of $b$ shall be chosen in such a way that $W(x, t)$ is a supersolution in $0<r<\frac{2}{n}, \quad 0 \leq t \leq \tau$ :

$$
W_{t} \geq \frac{m-1}{m}|\nabla W|^{2}+c \cdot \nabla W \frac{1}{m-1},
$$

and considering that:

$$
W_{t}=a b ; W_{r}=a,
$$

the following value for $b$ is obtained:

$$
b \geq \frac{m-1}{m} a+c \frac{1}{m-1}
$$

For the values of $a$ and $b$ in expressions (4.93) and (4.97) respectively, the function $W(x, t)$ is a supersolution locally:

$$
W(x, t) \geq w(x, t), \quad 0<|x|<\frac{2}{n}, \quad 0 \leq t \leq \tau .
$$

The inequality (4.98) permits to conclude that any local supersolution satisfies the null criteria in $B_{R}^{T}$, then, any minimal solution $w(x, t)$ satisfies such null criteria in $B_{R}^{T}$. 
The expression (4.70) provides the characteristic curve in relation with the diffusion front (note the parameter $m$ ) and the advection. Once the invaded specie starts the desertion in the direction of $c$, it follows a curve in $(x, t)$ given by (4.70). The invasive movement concentrates in the same trajectory to reduce the invaded population that follows an exponential decay. Furthermore, the existence of a finite propagation suggests that during the desertion, the diffusion is still relevant in the proximity of the null solution (i.e. the invaded tail). This can be interpret as the existence of a random movement of invaded specie in the tail where the invasive influence is negligible.

\section{Conclusions}

The proposed problem $P(1.4)$ has been discussed stressing aspects related with regularity, existence, uniqueness, comparison and behaviour of minimal and maximal solutions. In addition, the finite speed of propagation, induced by the porous medium diffusion, has been shown for $m>2$ and a characterization of such property has been explored. The propagation features of the specie $v$ when approaching the null solution have been shown. To this end, an exponential decreasing tail has been proved to exist moving along a characteristic curve in $(x, t)$. The invaded specie trajectory, to escape from the invasive, is given by the mentioned characteristic curve. Even when the invaded desertion is mainly governed by the advection, the non-linear diffusion still acts in the tail leading to a finite speed propagating front.

As a future research topic, it shall be precisely determined the relation between the exponential decay and the finite propagation speed. For this purpose, a travelling waves approximation together with the analysis of asymptotic trajectories in the phase plane [18] may constitute a method to explore. 


\section{References}

[1] Aguirre, J. and Escobedo, M. (1993). On the blow-up of solutions of a convective reaction diffusion equation. Proc. R. Soc. A, 123A, 433-460.

[2] Aguirre, J. and Escobedo, M. (1987). A Cauchy problem for $u_{t}-\Delta u=u^{p}$ with $0<p<1$. Asymptotic behaviour of solutions. Ann. Fac. Sci. Toulouse, Vol VIII, number 2.

[3] Ahn J., Yoon C. (2019) Global well-posedness and stability of constant equilibria in parabolic-elliptic chemotaxis system without gradient sensing. Nonlinearity, 32, pp. 13271351.

[4] Alford J.G. (2019) Mathematical Models Can Predict the Spread of an Invasive Species. In: Sriraman B. (eds) Handbook of the Mathematics of the Arts and Sciences. Springer, Cham. https://doi.org/10.1007/978-3-319-70658-052-1

[5] Banani, R.; Sankar K. R. and Biswas M. H. A. (2017). Effects on prey-predator with different functional responses. Int. J. Biomath. 10:08.

[6] Barenblatt (1996). Scaling, self-similarity, and intermediate asymptotics. Cambridge Texts in Applied Mathematics.

[7] Bedrossian J., Rodríguez, N. and Bertozzi, A.(2001). Local and global wellposedness for aggregation equations and patlak-keller-segel models with degenerate diffusion. Nonlinearity, $24(6): 1683$.

[8] Bertozzi, A., Slepcev, D. (2009). Existence and uniqueness of solutions to an aggregation equation with degenerate diffusion. Commun. Pure Appl. Anal. 9(6):1617.

[9] Bertsch, M. and Hilhorst, D. (1986) A density dependent diffusion equation in population dynamics: stabilization to equilibrium. SIAM J. Math. Anal. 17(4):863-883.

[10] Bhatti, M.; Zeeshan, A.; Ellahi,R.; Anwar Bég, O.; Kadir, A. (2019). Effects of coagulation on the two-phase peristaltic pumping of magnetized prandtl biofluid through an endoscopic annular geometry containing a porous medium, Chin. J. Phys. 58, 222-23. https://doi.org/10.1016/j.cjph.2019.02.004.

[11] Cho E., Kim Y.J.(2013). Starvation driven diffusion as a survival strategy of biological organisms Bull. Math. Biol., 75, pp. 845-870.

[12] De Pablo, A. (1989). Doctoral Thesis. Estudio de una ecuación de reacción - difusión Universidad Autónoma de Madrid.

[13] De Pablo, A.; Vázquez, J.L. (1991). Travelling waves and finite propagation in a reactiondiffusion Equation. J Differ Equ. 93 pp 19-61.

[14] Díaz, J. (2020). Modeling of an aircraft fire extinguishing process with a porous medium equation. SN Appl. Sci. 2, https://doi.org/10.1007/s42452-020-03891-9

[15] Díaz, J.L., Fernández M. (2020). Non-linear parabolic predator prey coupled system with convections. Int. J. Biomath. DOI https://doi.org/10.1142/S1793524521500054

[16] Ellahi, R., Hussain, F., Ishtiaq, F. et al. (2019) Peristaltic transport of Jeffrey fluid in a rectangular duct through a porous medium under the effect of partial slip: An application to upgrade industrial sieves/filters. Pramana - J Phys 93, 34. https://doi.org/10.1007/s12043019-1781-8

[17] Escobedo, M. and Herrero, M. (1991). A uniqueness result for a semilinear reaction-diffusion system. Proc. Am. Math. Soc. 112, 1.

[18] Ferreira, R.; de Pablo, A.; Reyes, G.; Sánchez, A. (2006) The interfaces of an inhomogeneous Porous Medium Equation with Convection. Commun. Partial. Differ. Equ. 31. 497-514.

[19] Haiyin Li. (2019). Hopf Bifurcation of Delayed Density-Dependent Predator-Prey Model. Acta Math. Sci. Series A, 39(2): 358-371. 
[20] Huang C.; Zhang, H.; Cao, J. and Hu, H. (2019). Stability and Hopf Bifurcation of a Delayed Prey-Predator Model with Disease in the Predator. Int J Bifurcat Chaos.Vol. 29, No. 07. https://doi.org/10.1142/S0218127419500913

[21] Jones, B.F. (1963). Singular integrals and parabolic equations. American Mathematical Society. United States of America.

[22] Keller E.F., Segel L.A. (1971). Traveling bands of chemotactic bacteria: a theoretical analysis. J. Theoret. Biol. 30, pp. 235-248.

[23] Mooney, H., Williamson, M. (2010). The problem of Biological Invasions. Oxford Scholarship.

[24] Pal, S.; Sasmal, S. K. and Pal, N. (2018). Chaos control in a discrete-time predator-prey model with weak Allee effect. Int. J. Biomath. Vol. 11, No. 07. https://doi.org/10.1142/S1793524518500894

[25] Pao, C. (2012). Nonlinear Parabolic and Elliptic Equations. Springer Science+Bussiness Media. North Carolina, United States of America.

[26] Shahid, A.; Huang, H.; Bhatti, M.M.; Zhang, L.; Ellahi, R. (2020). Numerical Investigation on the Swimming of Gyrotactic Microorganisms in Nanofluids through Porous Medium over a Stretched Surface. Mathematics, 8, 380. https://doi.org/10.3390/math803038

[27] Shuyan Qiu, Chunlai Mu, Xinyu Tu, (2020). Global dynamics of a two-species chemotaxisconsumption system with signal-dependent motilities, Nonlinear Anal Real World Appl. Volume 57, 103190,

[28] Szymańska, Z.; Rodrigo, C.; Lachowicz, M.; Chaplain, M. (2009). Mathematical modelling of cancer invasion of tissue: The role and effect of nonlocal interactions. Math Models Methods Appl Sci. 19. DOI: 10.1142/S0218202509003425.

[29] Tao Y., Winkler M.(2017) Effects of signal-dependent motilities in a keller-segel-type reactiondiffusion system. Math. Models Methods Appl. Sci., 27, pp. 1645

[30] Vázquez, J.L. (2006). The Porous Medium Equation, mathematical theory. Oxford Mathematical Monographs.Oxford.

[31] Volpert, V., Petrovskii, S. (2009). Reaction-diffusion waves in biology. Phys. Life. 6, pp 267310.

[32] Yoon C., Kim Y.J. (2016) Global existence and aggregation in a keller-segel model with fokkerPlanck diffusion. Acta Appl. Math., 149, pp. 101.

[33] Zhang, Y (2019). Degenerate Diffusions with Advection. Doctoral Thesis. University of California.

[34] Zu, L., Jiang, D. O'Regan, D. (2019). Periodic Solution for a Stochastic Non-autonomous Predator-Prey Model with Holling II Functional Response. Acta Appl Math 161, 89-105. doi.org/10.1007/s10440-018-0205-y 